\title{
THE INFRARED GRANULATION: OBSERVATIONS
}

\author{
SERGE KOUTCHMY \\ Institut d'Astrophysique, CNRS, 98 bis, Boulevard Arago, F-75014 Paris, France \\ and \\ National Solar Observatory, Sunspot, NM 88349, U.S.A.
}

\begin{abstract}
High spatial resolution observations of the solar granulation up to $3 \mu \mathrm{m}$ are possible on existing vacuum solar telescopes. They permit the analysis of the deepest photospheric layers near $1.7 \mu \mathrm{m}$. A high photometric accuracy is achieved using scanning techniques with a pinhole photometer; imaging methods are used for 2D-analysis. Statistically significant results on granulation, including power spectrum and histogram analysis, center-limb variations and lifetime analysis, are presented. Temperature fluctuations of periods near 5 minute are considered at 1.7 $\mu \mathrm{m}$, as well as large-scale variations at the scale of the meso- and, especially, the super-granulation. We also discuss the "abnormal" granulation in magnetic regions and the umbral granulation in the cores of sunspots.
\end{abstract}

Key words: infrared: stars - Sun: granulation - Sun: photosphere - sunspots

\section{Introductory Remarks}

\subsection{Present View of Solar Granulation}

Several reviews recently appeared on the subject, coming from observers, see Muller (1989), Karpinsky (1990), Title et al. (1990), Topka and Title (1991) and from theoreticians, see Chan et al. (1991). The book of Bray et al. (1984) is still a good introduction to the subject. Magneto-convection is considered by Weiss (1990). None of these works specifically considered the IR granulation, although a series of results were already presented, see Turon and Léna (1973), Albregtsen and Lynne Hansen, (1977), Worden (1975) and more recently Koutchmy (1990), hereafter referenced K90. The mentioned reviews by observers concentrate on the excellent optical observations and imaging of Pic du Midi and La Palma Observatories, results from the analysis of the Soviet stratoscope experiment of Pulkovo Observatory or of the SOUP experiment (seeing-free) on SpaceLab 2. They raise problems such as the following: Is granulation "turbulent" on small scales (R. Muller)? What is the true time variation of granules when the oscillatory component near 5 minutes is removed (Title et al.)? Is granulation well described by the network of dark lanes (V. Karpinsky) and what is the role of the (weak?) magnetic field? Where are shocks predicted (Cattaneo et al., 1989) in numerical simulations? What are the temperature fluctuations corresponding to the observed meso- and super-granulation? Optical granulation was also extensively observed at the VTT at Sacramento Peak Observatory by J. Evans, J. Beckers, R. Dunn, S. Keil and many others; here we shall concentrate on quantitative results obtained there in the IR, over the last 17 years. Many IR images, and even processed movies, have been obtained and are reported elsewhere in the present proceedings.

\subsection{Why Observe the IR Granulation? - a Theoretical Point of View}

The radiative opacity of the solar atmosphere reaches its absolute minimum in the $1.65 \mu \mathrm{m}$ spectral region of the continuum, see Figure 1. Obviously the $1.7 \mu \mathrm{m}$ 


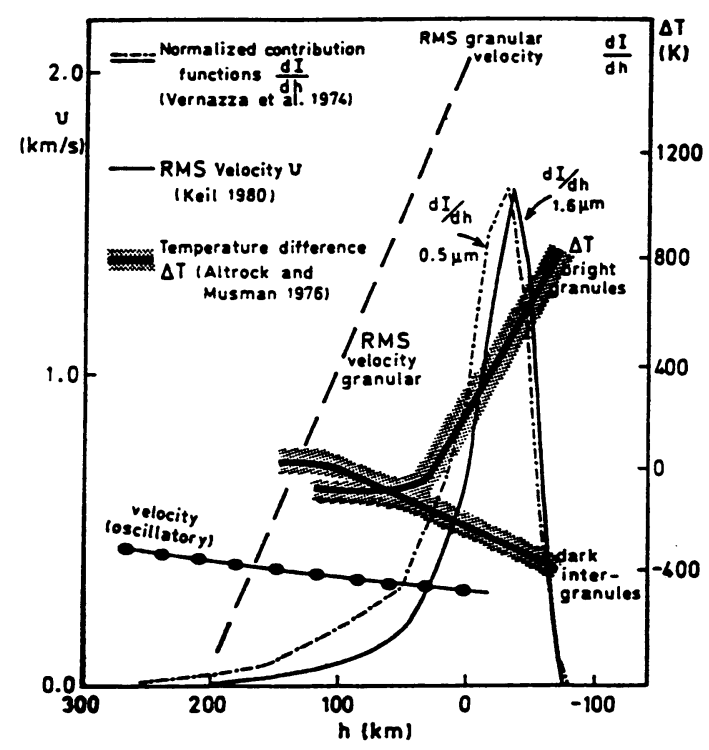

Fig. 1. Contribution functions for the wavelength regions $0.5 \mu \mathrm{m}$ and $1.6 \mu \mathrm{m}$, after Vernazza et al. (1974). On the same height scale are shown the behaviour of the temperature fluctuations from the granulation model of Altrock and Musman (1976) model and the RMS granular velocity and oscillations from Keil's (1980) analysis.

region of the opacity minimum region (OMR) is the most interesting for studying the layers which are most affected by the convection: Not only do we see deeper, we also partially avoid layers affected by 5 -minute oscillations, and layers where the temperature contrast shows an inversion, see Figure 1. Granular convective velocities seem to increase drastically when interpolated toward deeper regions Keil (1980) - so granules are expected to be seen in a more dynamical regime: shorter lifetimes and/or smaller size. Note also that the amplitude of 5-minute oscillations decreases with depth and, accordingly, convective motions are better measured in the OMR. Finally, because of the spectral behaviour of the Planck function, the interpretation of measured intensity fluctuations is made more easily in IR than in optical regions, when layers of different temperatures are considered, see Figure 2. Not only does a mixing of hot and cool gas along the line of sight occur in the optical regions, but the phenomenon is non-linear in terms of temperature, the main thermodynamical parameter; this is especially misleading when images are considered. Direct images of granulation in the OMR are published in K90 ; another example is given on Figure 2.

\subsection{Advantages and Disadvantages of Studying IR Granulation}

From the beginning, IR measurements have been made photoelectrically (or thermoelectrically), with strictly linear detectors of large dynamic range, so mistakes made in the visible through the use of the photographic films, which are often poorly 


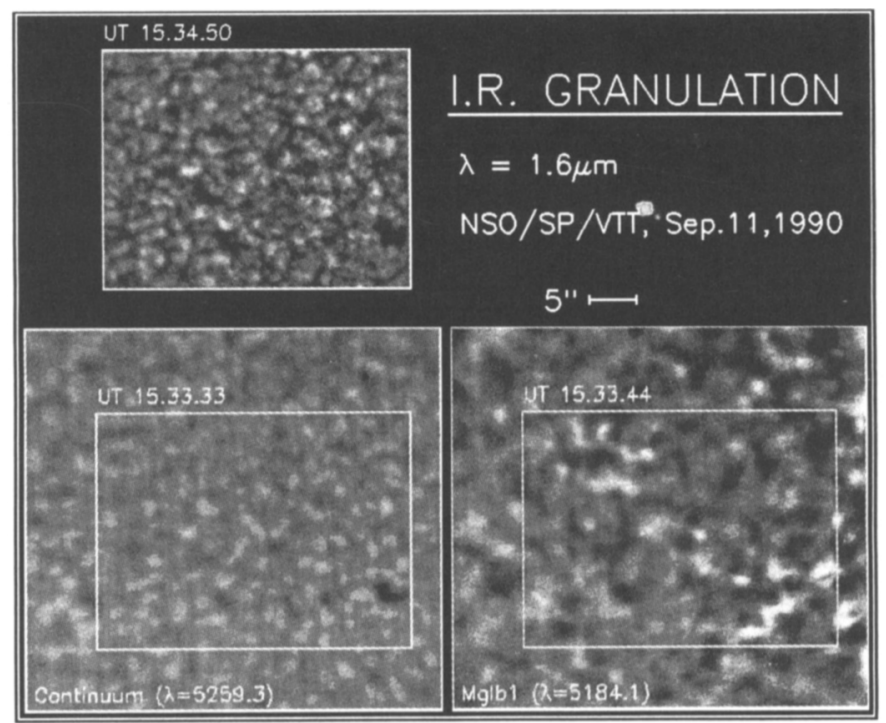

Fig. 2. Images of the solar granulation simultaneously observed in IR at $1.6 \mu \mathrm{m}$ in the OMR (top-left), and in the optical continuum at $525.93 \mathrm{~nm}$ (bottom-left) and the upper atmosphere at $518.41 \mathrm{~nm}$, with the UBF of the VTT of NSO/SPO. Note the good correlation between the IR and the optical granulation (separated by 77 seconds of time) and the lack of obvious correlation with magnetic elements shown in $\mathrm{Mg}$ b1 +0.4 (lower-right) evidenced by their bright emissions. The photographs were processed by F. Stauffer and T. Darvann at NSO/SPO.

calibrated, were avoided. Even the recently-performed observations in the visible with CCD cameras suffered from a lack of dynamic range (important for measuring the MTF of the telescope) and a limited field of view. We believe the combination of imaging techniques with scanning techniques yields a powerful and needed tool to extract good quantitative data for analyzing granulation.

Good photometric work requires the use of stable, well-controlled, observing conditions and the availability of good windows. In the IR, both the Earth's atmospheric transparency and the seeing are improved, sometimes dramatically, (at Sacramento Peak the transparency near $1.7 \mu \mathrm{m}$ was measured to be as high as $98 \%$ !) Windows available for observation of the solar continuum are also better than in the visible; line-blocking effects, which are especially severe in the blue region of the spectrum, are considerably reduced in IR. Moreover, the effect of the Earth's atmospheric differential refraction is also drastically decreased so that large spectral passbands, giving high signal-to-noise ratios, can be used for the fast imaging or scanning without losing spatial resolution (see Fig. 3). This advantage can be used to compensate for the disadvantage of losing the multiplex gain when scanning techniques are used with a single detector of large dynamical range - Koutchmy et al. (1977). 


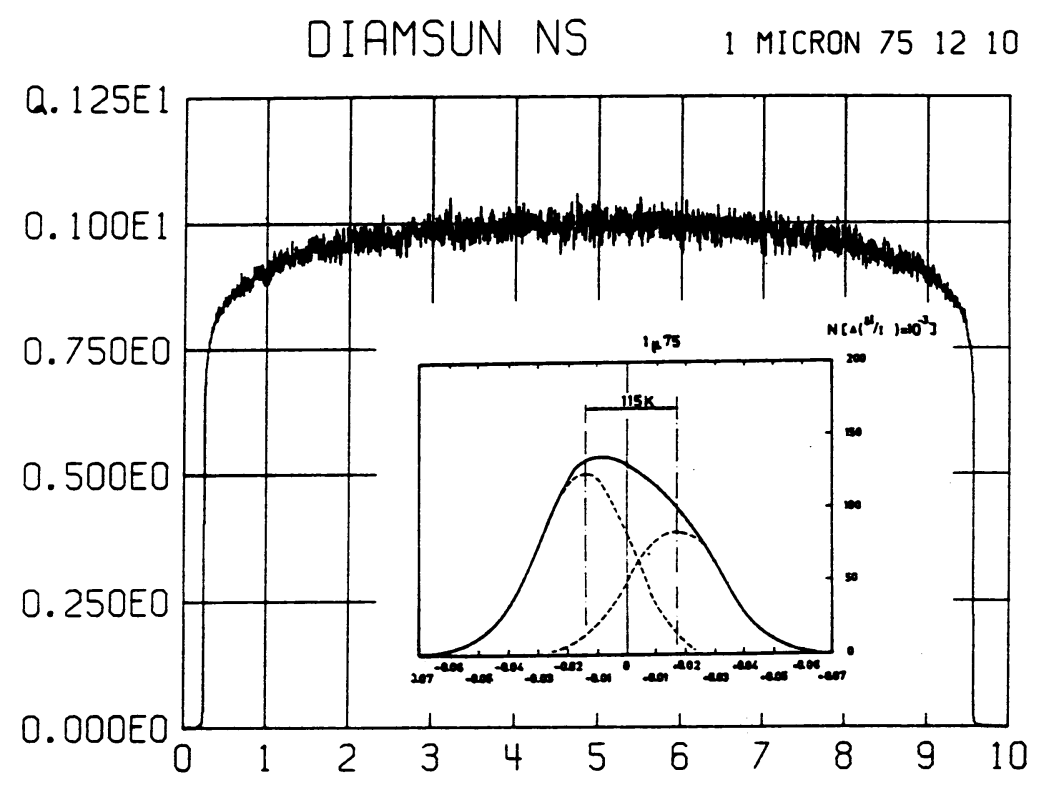

Fig. 3. A typical solar scan used to measure the RMS intensity variations of the IR granulation. The whole scan contains 11000 points. Note the level of noise, barely seen outside the limb, and the center-limb variation of the amplitude of the RMS fluctuations. The measured spatial resolution closely corresponds to the theoretical, when the combined effects of instrumental diffraction and the digitization step, with the 0.75 arcsec-diameter pin-hole, were taken into account. At the bottom, we have inserted the result of an histogram analysis of the central part of selected scans over an observing period of more than 2 hours. The histogram shows the largely skewed distribution in the intensities of IR granulation. Dark areas surpass bright areas by a ratio $1: 0.69$.

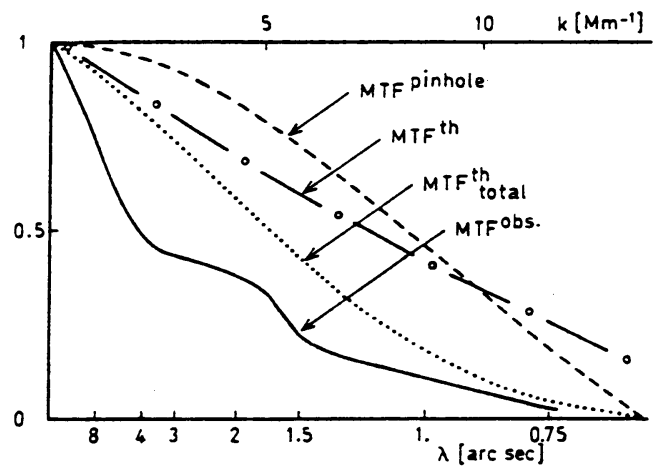

Fig. 4. Modulation Transfer Functions observed for different conditions, at the NSO/SP VTT near $1.7 \mu \mathrm{m}$. The total observed MTF was deduced from the analysis of many fast scans of the extreme-limb of the Sun, assuming a perfect step function for the external part of the solar limb. Theoretically computed MTF's are also shown. 
Up to now only telescopes designed for the optical region have been used to study the IR granulation; at $1.7 \mu \mathrm{m}$, such telescopes become diffraction-limited, because all aberrations are reduced by a factor 3 (see below). With respect to temporal variations, we find once again that working in the IR is more interesting. At a good, high-altitude, dry site, Earth-atmospheric induced effects are reduced, and indeed can easily be identified, because they are related to variations in microbarographic and water-vapor-content values.

The main disadvantage of working in the IR has been the lack of good 2Ddetectors; granulation images were made by scanning the Sun or by using very low quantum efficiency IR vidicons (see K90). In recent years the situation has completely changed, thanks to the appearance of new (not classified) highly-efficient 2D-detectors based on cooled $\mathrm{Hg}$-Cd-Te chips (see these proceedings), so we anticipate that many new results on IR granulation will soon appear. Finally, we note that intensity modulations produced by temperature fluctuations are considerably smaller at $1.7 \mu \mathrm{m}$ than in the visible. A $100 \mathrm{~K}$ temperature fluctuation corresponds to a $8.5 \%$ variation at $500 \mathrm{~nm}$ and only to $3.5 \%$ at $1.7 \mu \mathrm{m}$. This makes sunspot cores easier to study in the IR.

\section{Statistical Analysis of the IR Granulation}

\subsection{Amplitude of Small-Scale Granulation Intensity Fluctuations}

In 1975-76, we used a specially designed $\mathrm{PbS}$ pin-hole photometer to accurately measure intensity fluctuations over the solar disc observed at the prime focus of the VTT of NSO-SPO. The photometer used a chopper at $1100 \mathrm{~Hz}$ frequency and phase lock-in amplification, with a digitization rate of 100 point $\mathrm{sec}^{-1}$. A 14 bit/point precision A/D converter was used to record the data. Spectral selection was performed with a small IR f/60 monochromator with a $28 \times 28 \mathrm{~mm}^{2}$ grating blazed at $2 \mu \mathrm{m}$, and broad-band IR interference filters. For "fast" analysis we used only interference filters near $1.75 \mu \mathrm{m}$ and $2.2 \mu \mathrm{m}$; then the typical S/N ratio over the granulation scans was 2000 when a circular 0.75 arcsec diameter pin-hole was used (see Fig. 3). Only the very quiet Sun was considered in this analysis.

In this way, precise measurements of center-limb variations measurements were collected (see Koutchmy et al., 1977); the MTF of the telescope, including effects of scattered light, was accurately calibrated (see Fig. 4) by the use of fast scans of the extreme-limb. We computed the derivative of each scan to define the position of the inflection point and used only the external part of each scan to get the edge-like smearing function; taking the FFT of its derivative, we readily deduced the MTF, making the assumption that the true solar limb is far narrower than the FWHM of the line spread function. We found this method better than using the lunar limb at solar eclipses, because the lunar profile provides a rather poor knife edge.

An easy way to look at the observed FWHM of the smearing function is to analyze the auto-correlation function of the best granulation scans performed near the center of the Sun (see Fig. 5a); the half-width found from our best scans was of the order of 0.48 arcsec, which is the lowest value ever reported in the literature, including the visible region. This low value seems really to correspond to what 

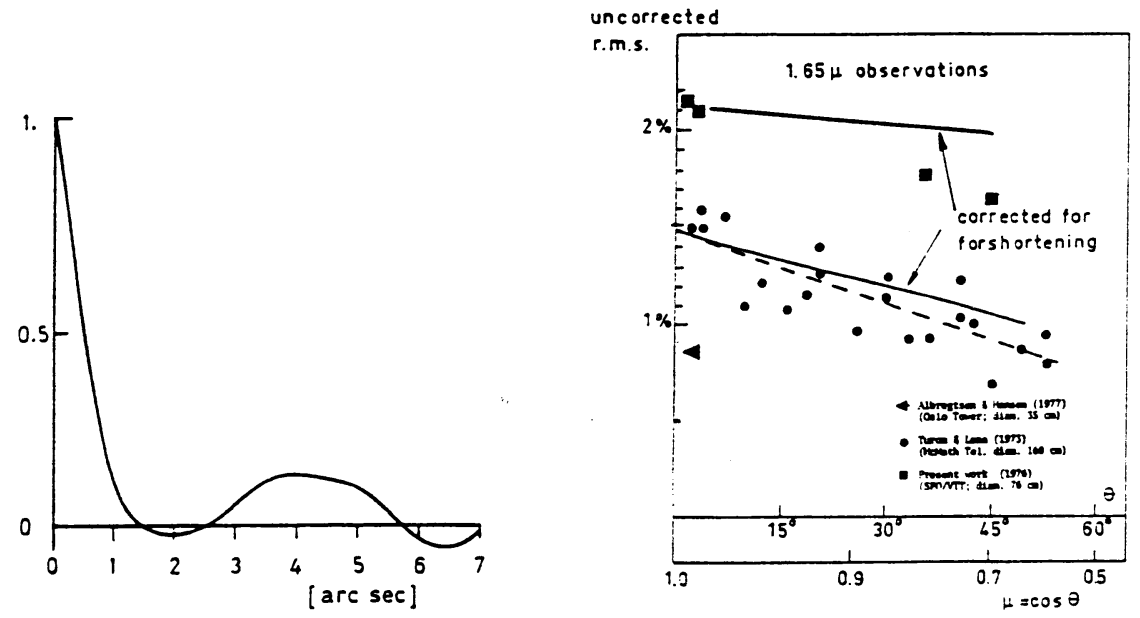

Fig. 5. a) Auto-correlation function computed for a selected 200 arcsec long scan taken near the center of the Sun, at $1.75 \mu \mathrm{m}$. The corresponding RMS was found equal to $2.17 \%$ and its width is $342 \mathrm{~km}$ or 0.476 arcsec. Note the minimum at 2 arcsec and the broad maximum around 4 arcsec. b) Center-limb variations of the RMS, calculated without corrections for the smearing. Data collected at NSO-SPO with high S/N ratio are the best.

we observe on the Sun in the OMR; it cannot be explained by the noise $(\mathrm{S} / \mathrm{N} \geq$ $2000)$ nor the value of the sampling resolution $(0.19 \operatorname{arcsec})$. It is an indication of the high quality of the data and suggests that the granulation in the OMR is possibly finer. The first indication that something is definitely different was given by the histogram analysis - see the inset at the bottom of Figure 3 . The skewness of the distribution is remarkable; the bi-distribution shows that areas occupied by darker than the average pixels dominate those of bright pixels in the ratio $1: 0.69$. In the visible, Keil (1977) obtained 0.88, and Karpinsky (1990) found 0.80 from high resolution stratoscope images. However, the bi-distribution shows an average difference of temperatures between dark and bright area of only $115 \mathrm{~K}$. This value should not be confused with that which could be deduced from the RMS of intensity fluctuations; Figure 5b shows our results compared to other published results, for different positions over the disk $(\cos \theta)$. The center-limb decrease is well apparent (after correction for foreshortening). These values are not corrected for the smearing shown by the measured MTF, Figure 4. After considering the spectral distribution of the IR granulation intensities (see further) we determined a correction factor of the order of 4.2 , so the corrected RMS at the disc center reaches $8.6 \%$ (instead of $2.05 \%$ ) at $1.75 \mu \mathrm{m}$; we should, however, confess that this value is affected by a large error bar due to the uncertainty of the correction at very small scales. 


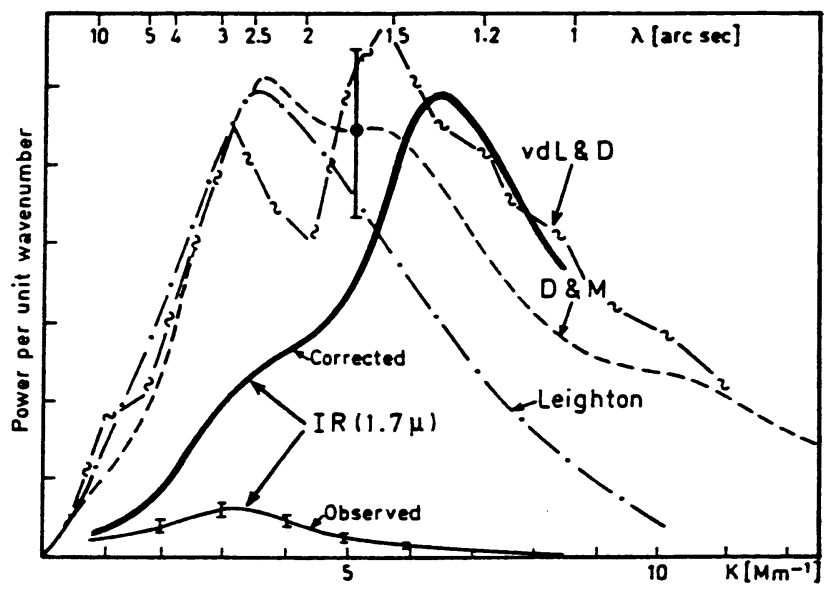

Fig. 6. Observed and corrected 2D spatial power spectra obtained in the IR (full lines). For comparison, we show results reported in visible light by different authors, over a 12-year interval, with improved resolution and correction methods: Leighton (1963), Deubner and Mattig (1975), and von der Lühe and Dunn (1987). Note that the power determinations in the visible extend to higher frequencies with improved methods; the relative lack of power at low frequencies in the OMR IR spectrum is striking.

\subsection{Power-Spectrum Analysis}

We selected the best scans performed at $1.75 \mu \mathrm{m}$ during a 3 -hour run, to perform a 1D power-spectrum analysis of very low noise, superposing several tens of spectra. The 1D average spectrum was readily transformed to a $2 \mathrm{D}$ power-spectrum by inverting the Abel transform (we also used the Hankel transform of auto-correlation functions like the one of Fig. 5a); spectra were smoothed to increase the level of confidence. A 2D power-spectrum per unit surface was published, without correction for the MTF, in K90, to show the difference in behaviour of raw data, between the visible light and the IR power spectra. In Figure 6 we show our best $2 \mathrm{D}$ spectrum before and after correction using the "observed" MTF of Figure 4. For comparison, Figure 6 also shows 2D power spectrum obtained by different authors at different epochs, in the visible. There is a clear tendency to report more and more power at higher frequencies; in the IR the dominance of power at high frequencies is more pronounced, bringing more arguments in favor of turbulent convection, see Muller (1989).

\subsection{Temporal Analysis: lifetimes; Temporal Fluctuations}

Using the scanning method (see Fig. 3), we analyzed the time variations of intensity fluctuations at granular size scales near the center of the Sun. Power spectrum analysis of a temporal sequence 46 minutes in length showed relative power in the 


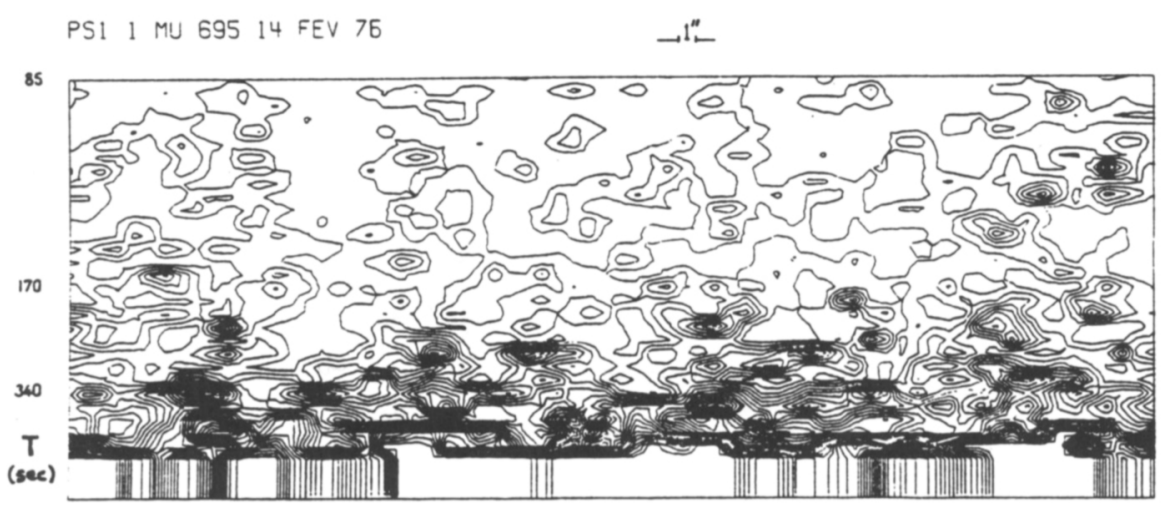

Fig. 7. Distribution of the power in temporal IR intensity variations spectra computed along a single direction near the center of the Sun. The cut-off frequency corresponds to $11.7 \mathrm{mHz}$ ( $\mathrm{T}=85$ second) and the time sequence is of 47 minute length.

5 -minute range at high spatial resolution but considerably less power when a 200 arcsec average is used (see K90). In Figure 7, curves of iso-power obtained at high spatial resolution are shown along one direction near the center of the Sun. Peaks of power are identified at the scale of the granulation from 3 to 6 minutes period. It is, however, difficult to distinguish between true recurring convection phenomena and oscillatory cells, so we should keep this in mind when looking at the problem of the lifetime of granules.

We tried to compute the lifetime of IR granules using $1 \mathrm{D}$ scans and crosscorrelations; looking at the decrease of the maximum amplitude of the cross-correlation function computed with different time lags we found a rather short lifetime, of the order of 3.3 minute for the decrement of the best fit of the data to an exponential function (lifetime should be considered equal to at least twice the value of the decrement). Figure $8 \mathrm{a}$ illustrates the typical cross-correlation function we obtained with a 160-second time lag. Note the shift of the bisector of the function, which corresponds to the shift produced by solar rotation, seen by the displacements of granules in the $\mathrm{E}-\mathrm{W}$ direction. The rather short lifetime found in this analysis is attributed to the dominance of very small granules, partially to fragments of granules, which cause a loss of correlation using this method; oscillations could possibly also influence the results. In 1988-90 several movies of IR granulation were obtained (see K90). A movie of 45 minutes of continuous observations was processed by $\mathrm{T}$. Darvann (these proceedings). Using the filtered and selected images of this movie, correlation coefficients between images were computed - see Figure $8 \mathrm{~b}$. Now the decrement of the average curve corresponds to 7.5 minute (lifetime 15 minute), which seems rather long. The processing which has been applied to permit a precise tracking, to compute the flow map, seems to favor longer-ifetime granules and possibly agglomerates of granules and cell-like structures with with a 

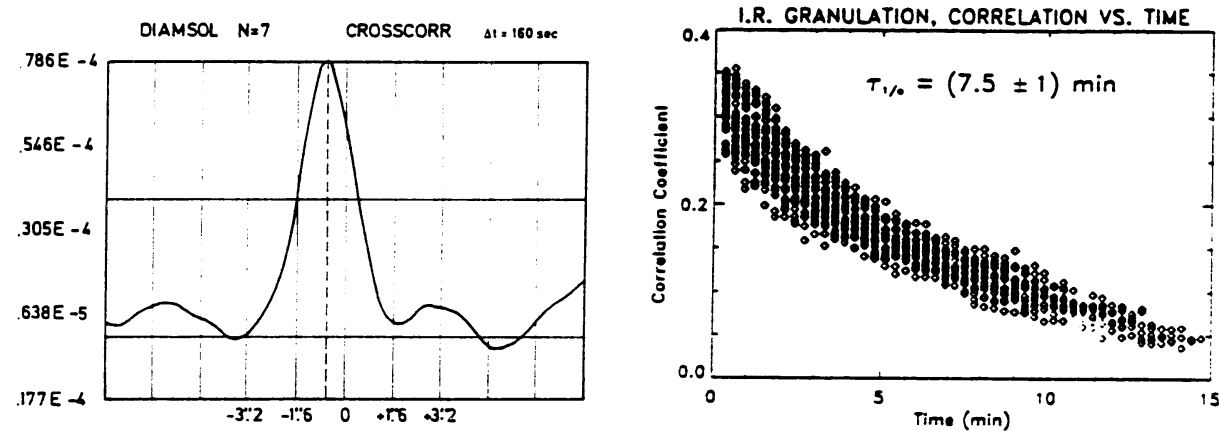

Fig. 8. a) Typical cross-correlation function obtained using two 1D scans made near the equator at $1.75 \mu \mathrm{m}$. The time lag is 160 second; note the shift due to solar rotation. b) Lifetime of granules computed by $T$. Darvann from a processed 45 -minute movie at $1.6 \mu \mathrm{m}$.

longer lifetime. We conclude that both 6- and 15-minute lifetimes are statistically significant, depending of the size of granules.

\section{Large-Scale Granulation}

\subsection{Statistical Analysis}

Sizes of convective cells are not limited to the granulation size, from about 1 to 3 arcsec. In the visible, both Doppler shifts and intensity modulations reveal cells at a meso-granulation size, between 5 and $40 \mathrm{arcsec}$, and also at the supergranulation (SG) size near 42 arcsec. A temperature contrast of the order of $20 \mathrm{~K}$ was found by Koutchmy and Lebecq (1986) for meso-granules, after eliminating 5-minute oscillations. In the OMR, at $1.7 \mu \mathrm{m}$, Worden (1975) and Koutchmy (1978) gave the first results on the SG. We analysed a $940 \times 940 \operatorname{arcsec}^{2}$ matrix of intensity fluctuations, see Figure 3, taken over the central part of a very quiet Sun $($ Oct.11, 1975) at 1.7 $\mu \mathrm{m}$. After accurately removing the center-to-limb variations of normalized scans, we performed a $1 \mathrm{D}$-autocorrelation analysis $10^{3}$ times and looked at spacings near the size of $\mathrm{SG}$ - see the resulting curve in Figure $9 \mathrm{a}$. For sizes near $27 \mathrm{Mm}$, the autocorrelation function is definitively positive; only at $36 \mathrm{Mm}$ a slightly negative value is found; again near $55 \mathrm{Mm}$ a large maximum is observed. From this analysis we conclude that the boundaries of SG are definitely bright, statistically speaking - see Figure 9a - contrary to the first results reported by Worden (1975), which were made with a rather low $\mathrm{S} / \mathrm{N}$ ratio through the large dispersion spectrograph of the $80-\mathrm{cm}$ Kitt Peak auxiliary heliostat. A positive contrast at SG size is also reported in Lin and Kuhn (1992); it is obviously related to the magnetic nature of the boundaries of the SG. To estimate the temperature modulation at the bound- 

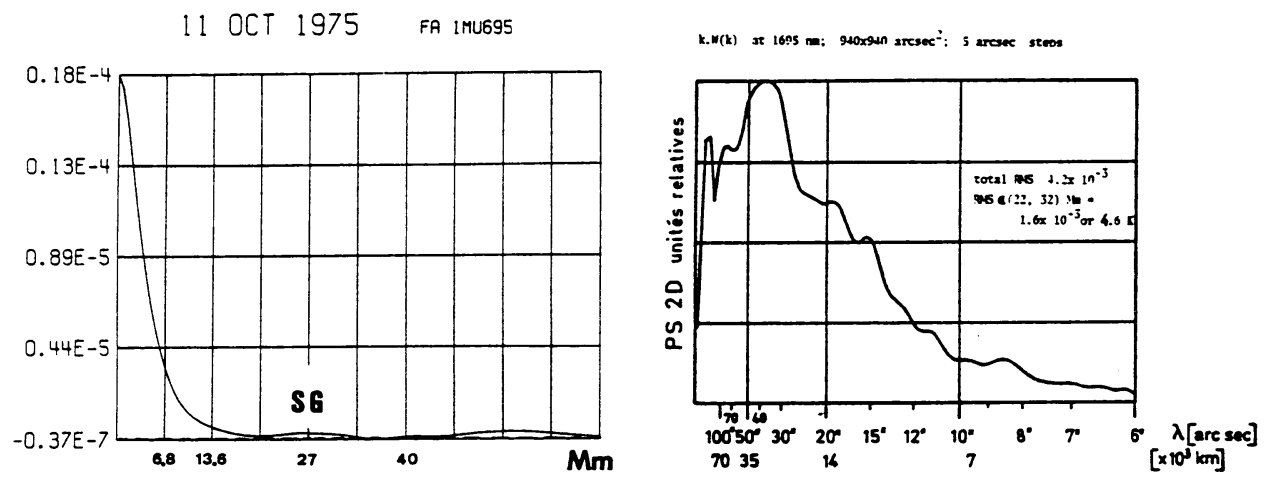

Fig. 9. a) Superposed autocorrelation function of the central part of the quiet Sun to show the positive temperature contrast observed at the super-granulation size. b) The corresponding $2 \mathrm{D}$-Power spectra per unit of wavenumber. The main peak shows a SG size of around 42 arcsec.

ary of SG, we used the 2D power-spectrum of Figure $9 \mathrm{~b}$ showing a total RMS of $4.2 \times 10^{-3}$. The power measured between 22 and $32 \mathrm{Mm}$, which includes the main peak corresponding to the $\mathrm{SG}$, gives an RMS temperature contrast of $4.6 \mathrm{~K}$; because 5 -minute oscillations are not fully eliminated in this analysis, the true contrast is probably smaller but still positive.

\subsection{Mesogranulation Flow}

Using visible granules as tracers, L. November (1986) showed how it is possible to compute a flow map which reveals the mesogranules. The 45-minute movie obtained in 1988 at $1.6 \mu \mathrm{m}$, (see $\mathrm{K} 90$ ), has been processed with this method. T. Darvann, (1992), performed a full analysis of the movie with very interesting results. The mesogranulation flow, and probably the SG flow, are well evidenced, see Figure 10; boundaries are well represented by the positions of "corks" which follow the flow after more than 8 hours. A very interesting deep sink appears in the field of view and boundaries show a kind of sheared flow. Several meso-scale cells are seen on the divergence map - see the contours in Figure 10. Contrary to the divergence, a vorticity-map apparently shows large changes over a time lag of 20 minutes, reversing its sign in several locations; this behaviour suggests the presence of torsional waves at meso-scales with periods of 20 to 40 minutes. More data are needed to confirm this very interesting finding coming from the analysis of the 1.6 $\mu \mathrm{m}$ OMR granulation movie. An additional analysis, which is needed in the future, is a comparison with the map of magnetic elements of the photosphere, which are presumably located where the flow converges. The presence of meso-scale torsional 


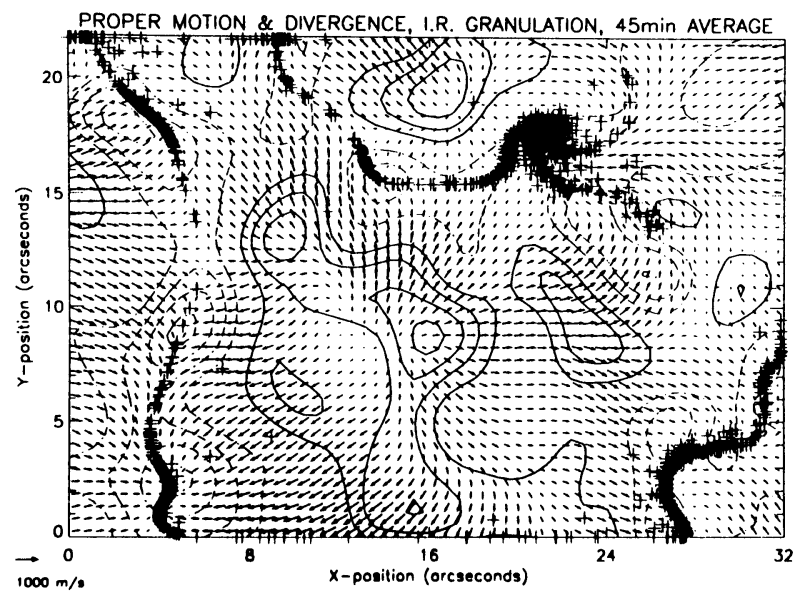

Fig. 10. Map of the flow deduced from the 45-minute granulation movie of 1988 obtained in the OMR. Contours show divergence of the average flow, solid line positive, dashed line negative (downflow). Cork positions after 8 hours are superposed to show the boundaries of a SG cell; note also several meso-scale cells inside the SG.

waves, with 20 to 40 minute periods, would be of great importance in enhancing magnetic-field effects in higher layers, up to the transition region.

\section{Discusion and Conclusions}

\subsection{IR Granulation and the Magnetic Field}

Examples of what granulation phenomena observed in the OMR can bring to the analysis of the origin of small magnetic elements are shown in Figs. 2 and 10; Figure 9 a shows statistically the effect of concentration of magnetic elements at the boundaries of SG of the quiet Sun: these produce an excess of temperature (network bright point) and they occur at the locations of converging large scale flows. Note that we are referring to magnetic elements outside active regions, avoiding pores and pore-like structures which, obviously, show a negative contrast (see Fig. 2 and the paper of Darvann and Koutchmy in the present proceedings).

In regions of large concentration of magnetic field, namely the cores of sunspots, umbral granulation or umbral dots are observed. At 1.6 and $2.2 \mu \mathrm{m}$, we observed some sunspots by scanning, and also by imaging with diffraction-limited resolution at video speed. Umbral dots are easily seen; the images are stable and because the average contrast of the core is of the order of 0.6 or 0.7 - see Kotov and Koutchmy (1992) - there is no problem of scattered light as in the visible. We believe that the bright IR umbral dots will be more seriously analysed in the future, permitting new insights into the problems of magneto-convection and the origin of sunspots. Not only is the problem of spurious scattered light avoided, but magnetic effects 
are better separated from thermal and velocity effects; and the seeing is improved.

\subsection{Concluding Remarks}

Observing in the OMR has provided new results on granulation:

i) a large spectrum of cells, including the smallest at sub-arcsecond size, is observed;

ii) Convective phenomena are well observed with different spatial and temporal scales; there is now a need for line-profile analysis with high spatial resolution;

iii) The best spatial resolution is achieved in the OMR; simultaneous observations at two or more wavelengths are entirely feasible for a better coverage in heights.

Existing and planned solar telescopes should be adapted to IR needs and new ground-based facilities can be envisaged in the near future, based on new technology designs for building good IR telescopes.

\section{References}

Albregtsen, F. and Lynne Hansen, T.: 1977, Solar Phys. 54, 31.

Altrock, R.C. and Musman, S.: 1976, Astrophys. J. 203, 533.

Bray, R.J., Loughhead, R.E. and Durrant, C.J.: 1984, The Solar Granulation, Cambridge University Press.

Cattaneo, F., Hurlburt, N.E. and Toomre, J.: 1989, in R. J. Rutten and G. Severino (eds.), Solar and Stellar Granulation, Kluwer Academic Publishers, Dordrecht, p. 415.

Chan, K.L., Norland, A., Steffen, M. and Stein, R.F.: 1991, in A. Cox, W. Livingston, and M. Matthews (eds.), Solar Interior and Atmosphere, University of Arizona Press, Tucson, Arizona, p. 223.

Darvann, T. and Koutchmy S.: 1993, these proceedings.

Deubner, F.L. and Mattig, W.: 1975, Astron. Astrophys. 45, 167.

Foukal, P., Little, R., Graves, J., Rabin, D. and Lynch, D.: 1990, Astrophys. J. 353, 712.

Karpinsky, V.N.: 1990, in J. Stenflo (ed.), 'Solar Photosphere: Structure, Convection and Magnetic Fields', IA U Symp. 138, 67.

Keil, S.L.: 1977, Solar Phys. 53, 359.

Keil, S.L.: 1980, Astron. Astrophys. 82, 144.

Kotov, V. and Koutchmy, S.: 1993, these proceedings.

Koutchmy, S.: 1978, in "Pleins Feux sur la Physique Solaire", Proceedings of the $2^{\text {nd }}$ Europ. Ass. Solar Phys. Meeting, ed. CNRS, p. 155.

Koutchmy, S.: 1990, in J. Stenflo (ed.), 'Solar Photosphere: Structure, Convection and Magnetic Fields', IAU Symp. 138, 81.

Koutchmy, S., Koutchmy, O. and Kotov, V.: 1977, Astron. Astrophys. 59, 189.

Koutchmy, S. and Lebecq, C.: 1986, Astron. Astrophys. 169, 323.

Leighton, R.B.: 1983, Ann. Rev. Astron. Astrophys. 1, 69.

Lin, K. and Kuhn, J.R.: 1992, Solar Phys. , in press.

Muller, R.: 1989, in R. J. Rutten and G. Severino (eds.), Solar and Stellar Granulation, Kluwer Academic Publishers, Dordrecht, p. 101.

November, L.J.: 1986, Appl. Optics , 25, 3, 392.

Title, A.M., Shine, R.A., Tarbell, T.D., Topka, K.P. and Scharmer, G.B.: 1990, in J. Stenflo (ed.), 'Solar Photosphere: Structure, Convection and Magnetic Fields', IA U Symp. 138, 49.

Topka, K.P. and Title, A.M.: 1991, in A. Cox, W. Livingston, and M. Matthews (eds.), Solar Interior and Atmosphere, University of Arizona Press, Tucson, Arizona, p. 727.

Turon, P.J.: 1975, Solar Phys. 41, 271.

Turon, P.J. and Léna, P.: 1973, Solar Phys. 30, 3.

Vernazza, J.E., Avrett, E.H. and Loeser, R.: 1981, Astrophys. J. Suppl. 45, 635.

von der Lühe, O. and Dunn, R.B.: 1987, Astron. Astrophys. 177, 265.

Weiss, N.O.: 1990, Proc. IA U Symp. 142, 139.

Worden, S.P.: 1975, Solar Phys. 45, 521. 